\title{
JONES AND $Q$ POLYNOMIALS FOR 2-BRIDGE KNOTS AND LINKS
}

\author{
TAIZO KANENOBU
}

(Communicated by Frederick R. Cohen)

Dedicated to Professor Yoko Tao on her sixtieth birthday

\begin{abstract}
It is known that the $Q$ polynomial of a 2-bridge knot or link can be obtained from the Jones polynomial. We construct arbitrarily many 2-bridge knots or links with the same $Q$ polynomial but distinct Jones polynomials.
\end{abstract}

The Jones polynomial $V_{L}(t) \in Z\left[t^{ \pm 1 / 2}\right]$ [5] is an invariant of the isotopy type of an oriented knot or link $L$ in the 3-sphere. The writhe $w(D)$ of an oriented planar diagram $D$ of $L$ is the sum of the signs at all the crossings of $D$, according to the convention explained in Figure 1 (p. 836). Let $|D|$ be a diagram $D$ with its orientation unknown. Then Kauffman's bracket polynomial $\langle D\rangle \in Z\left[A^{ \pm 1}\right][10]$ of $|D|$, which is a regular isotopy invariant, is defined by

$$
\begin{aligned}
\langle 0\rangle & =1 \text { for a simple closed curve } 0, \\
\left\langle D^{\prime}\right\rangle & =\left(-A^{2}-A^{-2}\right)\langle D\rangle, \\
\left\langle D_{ \pm}\right\rangle & =A^{ \pm 1}\left\langle D_{0}\right\rangle+A^{\mp 1}\left\langle D_{\infty}\right\rangle,
\end{aligned}
$$

where $\left|D^{\prime}\right|$ is the disjoint union of $|D|$ and a simple closed curve and $\left|D_{i}\right|$ are identical diagrams except near one point, where they are as in Figure 2 (p. 836). Then the Jones polynomial can be defined using the formula

$$
V_{L}\left(A^{4}\right)=\left(-A^{3}\right)^{-w(D)}\langle D\rangle \text {. }
$$

The $Q$ polynomial $Q_{L}(x) \in Z\left[x^{ \pm 1}\right][1,4]$ is an invariant of the isotopy type of an unoriented knot or link $|L|$ in the 3 -sphere, which is defined by the following formulas:

$$
\begin{aligned}
Q_{U}(x) & =1 \text { for the unknot } U, \\
Q_{L_{+}}(x)+Q_{L_{-}}(x) & =x\left(Q_{L_{0}}(x)+Q_{L_{\infty}}(x)\right),
\end{aligned}
$$

where the links $\left|L_{i}\right|$ have diagrams $\left|D_{i}\right|$ which are as in the above.

Received by the editors July $21,1989$.

1980 Mathematics Subject Classification (1985 Revision). Primary 57M25.

Key words and phrases. 2-bridge knot, 2-bridge link, $Q$ polynomial, Jones polynomial.

The author was partially supported by Grant-in-Aid for Encouragement of Young Scientists (No. 01740057), Ministry of Education, Science, and Culture. 


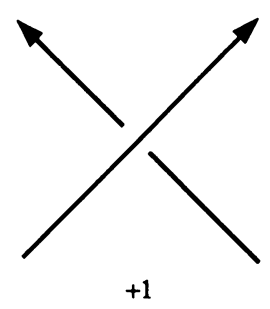

$+1$

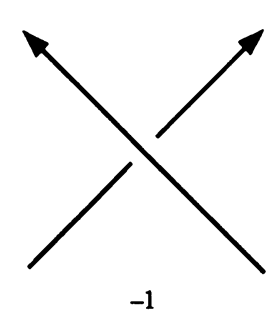

$-1$

FIGURE 1

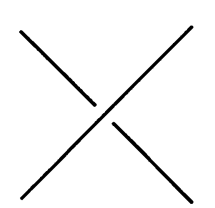

$\left|D_{+}\right|$

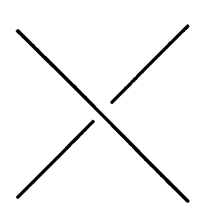

$\left|D_{-}\right|$

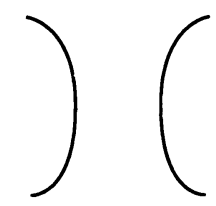

$\left|D_{0}\right|$

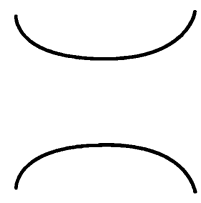

$\left|D_{\infty}\right|$

Figure 2

The author [7] discovered a relation between the Jones and $Q$ polynomials of a 2-bridge knot or link (see, for example, [2, Chapter 12]).

Theorem *. If $L$ is a 2-bridge knot or link, then it holds that

$$
Q_{L}(x)=2 x^{-1} V_{L}(t) V_{L}\left(t^{-1}\right)+1-2 x^{-1},
$$

where $x=-t-t^{-1}$. Identically,

$$
Q_{L}(x)=2 x^{-1}\langle D\rangle\langle D !\rangle+1-2 x^{-1},
$$

where $x=-A^{4}-A^{-4}, D$ is a diagram for $L$, and $D$ ! is a mirror image of $D$, so that $\langle D !\rangle(A)=\langle D\rangle\left(A^{-1}\right)$.

This theorem implies that the $Q$ polynomial of a 2-bridge knot or link can be deduced from the Jones polynomial. Conversely, even if a $Q$ polynomial of some 2-bridge knot or link is given, we cannot necessarily infer its Jones polynomial. In fact, through a computer calculation of polynomial invariants of 2-bridge knots and links [9], except for a reflection such as right- and lefthanded trefoils, we found many pairs of 2-bridge knots and links with the same $Q$ polynomial but distinct Jones polynomials; $\left\{10_{14}, 10_{31}\right\},\left\{10_{19}, 10_{36}\right\}$, and $\left\{9_{4}^{2}, 9_{10}^{2}\right\}$ are such pairs in the table of [15]. Also it is known that there exist arbitrarily many skein-equivalent 2-bridge knots [8] and links [6], which thus have the same 2-variable Jones, Jones, Alexander, and $Q$ polynomials. (See [12] for the definition of skein equivalence and 2-variable Jones polynomial.) 
Generalizing these examples, we prove

Theorem. For any positive integer $N$, there exist $N$ sets of $2^{N}$ 2-bridge knots (resp. links) $\mathscr{K}_{1}, \mathscr{K}_{2}, \ldots, \mathscr{K}_{N}$ with $\mathscr{K}_{i}=\left\{K_{i 1}, K_{i 2}, \ldots, K_{i 2^{N}}\right\}$ such that

(i) all the knots (resp. links) in the union $\bigcup_{i=1}^{N} \mathscr{H}$ share the same $Q$ and Alexander (resp. 2-variable Alexander) polynomials;

(ii) all the knots (resp. links) in each $\mathscr{K}_{i}$ are skein-equivalent, and so they have the same 2-variable Jones, Jones, and Alexander (resp. reduced Alexander) polynomials; and

(iii) all the knots (resp. links) $K_{11}, K_{21}, \ldots, K_{N 1}$ have mutually distinct Jones polynomials.

\section{Preliminaries}

Let $\alpha$ be a 3-braid. We denote a 3-braid $\alpha S_{2}^{n} \alpha^{-1}$ and $\alpha S_{2}^{m} \alpha^{-1} S_{1}^{n} \alpha, m$, $n \in Z$, by $\alpha(n)$ and $\alpha(m, n)$, respectively, where $S_{1}$ and $S_{2}$ are elementary 3-braids as shown in Figure 3. Let $G_{\alpha}$ and $H_{\alpha}$ be unoriented 2-bridge knot or link diagrams as shown in Figure 4. From [8, Proposition 2.4], we have

\section{Lemma 1.}

(i) $\left\langle H_{\alpha(n)}\right\rangle=A^{n}\left[d-\left\{1-\left(-A^{-4}\right)^{n}\right\} d^{-1}\left\langle G_{\alpha}\right\rangle\left\langle G_{\alpha} !\right\rangle\right]$,

(ii) $\left\langle G_{\alpha(m, n)}\right\rangle=A^{m+n}\left\langle G_{\alpha}\right\rangle\left[\left(-A^{-4}\right)^{m}+\left(-A^{-4}\right)^{n}-1+\left\{1-\left(-A^{-4}\right)^{m}\right\}\right.$

$$
\times\left\{1-\left(-A^{-4}\right)^{n}\right\} d^{-2}\left\langle G_{\alpha}\right\rangle\left\langle G_{\alpha} !\right\rangle,
$$

where $d=-A^{2}-A^{-2}$ is the bracket polynomial of a trivial 2-component link diagram without any crossing.

Let $Q_{\alpha}(x), Q_{\alpha(n)}(x)$, and $Q_{\alpha(m, n)}(x)$ be the $Q$ polynomials of unoriented 2-bridge knots or links with diagrams $G_{\alpha}, H_{\alpha(n)}$, and $G_{\alpha(m, n)}$, respectively.

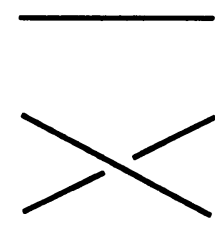

$S_{1}$

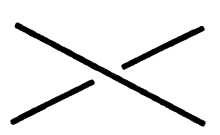

$S_{2}$

Figure 3 

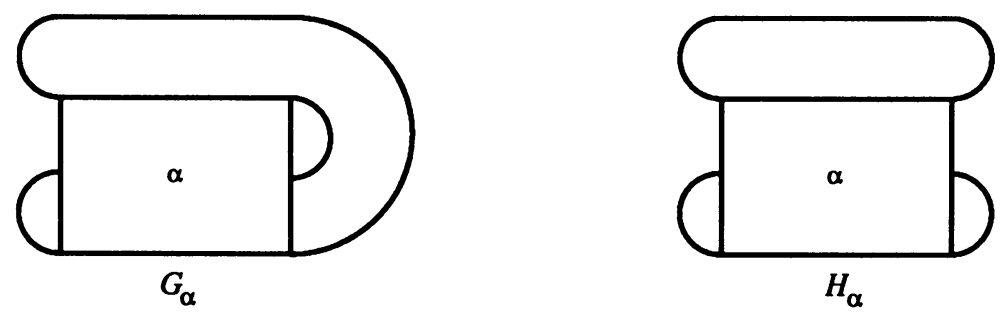

FIGURE 4

Then we have

\section{Lemma 2.}

(i) $Q_{\alpha(n)}(x)=\frac{1}{2}\left(\sigma_{n+1}-\sigma_{n-1}\right)\left(\mu-\mu^{-1} Q_{\alpha}(x)^{2}\right)+\mu^{-1} Q_{\alpha}(x)^{2}$,

(ii) $Q_{\alpha(1,-1)}(x)=\left(x^{2} / 4\right)\left(Q_{\alpha}(x)+1\right)^{2}\left(Q_{\alpha}(x)+\mu\right)-\mu$,

where $\sigma_{n} \in Z\left[x^{ \pm 1}\right]$ is the polynomial defined by $\sigma_{n-1}+\sigma_{n+1}=x \sigma_{n}, \sigma_{0}=0$, $\sigma_{1}=1$, and $\mu=2 x^{-1}-1$ is the $Q$ polynomial of a trivial 2-component link. Proof.

(i) is Lemma 6.1 of [6].

(ii) By Lemma 1 (ii),

$$
\left\langle G_{\alpha(1,-1)}\right\rangle=\left\langle G_{\alpha}\right\rangle\left(-A^{-4}-1-A^{4}+\left\langle G_{\alpha}\right\rangle\left\langle G_{\alpha} !\right\rangle\right)
$$

By substituting $\left\langle G_{\alpha}\right\rangle\left\langle G_{\alpha} !\right\rangle=\frac{x}{2}\left(Q_{\alpha}(x)+\mu\right)$ (Theorem $\left.{ }^{*}\right)$ and $A^{-4}+A^{4}=-x$, this becomes

$$
\left\langle G_{\alpha(1,-1)}\right\rangle=\frac{x}{2}\left\langle G_{\alpha}\right\rangle\left(Q_{\alpha}(x)+1\right)
$$

from which we have

$$
\left\langle G_{\alpha(1,-1)} !\right\rangle=\frac{x}{2}\left\langle G_{\alpha} !\right\rangle\left(Q_{\alpha}(x)+1\right) .
$$

Substituting these formulas into $\left\langle G_{\alpha(1,-1)}\right\rangle\left\langle G_{\alpha(1,-1)} !\right\rangle=\frac{x}{2}\left(Q_{\alpha(1,-1)}(x)+\mu\right)$ (Theorem *), we obtain (ii).

Using Lemma 2, we can prove the following by induction:

\section{Lemma 3.}

$$
\begin{aligned}
Q_{\alpha(m, n)}(x)= & \frac{1}{4 \mu^{2}}\left(\sigma_{m+1}-\sigma_{m-1}-2\right)\left(\sigma_{n+1}-\sigma_{n-1}-2\right) Q_{\alpha}(x)^{3} \\
& -\frac{1}{4} x(x+2) \sigma_{m} \sigma_{n} Q_{\alpha}(x)^{2} \\
& +\left\{1-\frac{1}{4}\left(\sigma_{m+1}-\sigma_{m-1}-2\right)\left(\sigma_{n+1}-\sigma_{n-1}-2\right)\right\} Q_{\alpha}(x) \\
& +\left(\mu^{2} / 4\right) x(x+2) \sigma_{m} \sigma_{n}
\end{aligned}
$$


Since $\sigma_{n+1}-\sigma_{n-1}=\sigma_{-n+1}-\sigma_{-n-1}$ and $\sigma_{m} \sigma_{n}=\sigma_{-m} \sigma_{-n}$, we have from this Lemma 4. Let $\alpha$ and $\beta$ be 3-braids. If $Q_{\alpha}(x)=Q_{\beta}(x)$, then $Q_{\alpha(m, n)}(x)=$ $Q_{\alpha(-m,-n)}(x)=Q_{\beta(m, n)}(x)=Q_{\beta(-m,-n)}(x)$.

Remark 1 . We can also prove the following by induction:

$$
\begin{aligned}
& \left(Q_{\alpha}(x)+\mu\right)\left(Q_{\alpha(m, n)}(x)+Q_{\alpha(m+n)}(x)\right) \\
& \quad=\left(Q_{\alpha(m)}(x)+Q_{\alpha}(x)\right)\left(Q_{\alpha(n)}(x)+Q_{\alpha}(x)\right) .
\end{aligned}
$$

Remark 2. Let $\alpha=S_{2}^{2} S_{1}^{2}$. Then the 2-bridge knots and links $10_{14}, 10_{31}$, $10_{19}, 10_{36}, 9_{4}^{2}, 9_{10}^{2}$ given above have diagrams $G_{\alpha(-2,1)}, G_{\alpha(2,-1)}, G_{\alpha(2,1)}$, $G_{\alpha(-2,-1)}, G_{\alpha(1,1)}, G_{\alpha(-1,-1)} !$, respectively.

Suppose that $\alpha$ is a pure 3-braid and $m, n$ are even integers. Let $\nabla_{\alpha}(z)$ and $\nabla_{\alpha(m, n)}(z)$ be the Conway polynomials of the 2-bridge knots with diagrams $G_{\alpha}$ and $G_{\alpha(m, n)}$, respectively. (See [3]. Substituting $z=t^{1 / 2}-t^{-1 / 2}$, we obtain the Alexander polynomials.) Then we can prove the following by induction:

Lemma 5. $\nabla_{\alpha(m, n)}(z)=\frac{m n}{4} \nabla_{\alpha}(z)^{3}+\nabla_{\alpha}(z)$.

Suppose that $\alpha$ is a pure 3-braid and $m, n$ are even integers. Let $\nabla_{\alpha}\left(t_{1}, t_{2}\right)$ and $\nabla_{\alpha(m, n)}\left(t_{1}, t_{2}\right)$ be the Conway potential function of the 2-bridge links with diagrams $H_{\alpha}$ and $H_{\alpha S_{1}^{m} \alpha^{-1} S_{1}^{n} \alpha}$, respectively, where the links are oriented so that they coincide if $m n=0$. (See [3]. Substituting $t_{i}$ for $t_{i}^{1 / 2}, i=1,2$, we obtain the 2-variable Alexander polynomial.) Then we can prove the following by induction:

\section{Lemma 6.}

$$
\nabla_{\alpha(m, n)}\left(t_{1}, t_{2}\right)=\frac{m n}{4}\left(t_{1}-t_{1}^{-1}\right)^{2}\left(t_{2}-t_{2}^{-1}\right) \nabla_{\alpha}\left(t_{1}, t_{2}\right)^{3}+\nabla_{\alpha}\left(t_{1}, t_{2}\right) .
$$

\section{Proof of THEOREM}

Knot Case. Let $\alpha=S_{2}^{2} S_{1}^{4}$ and $\varepsilon_{i} u_{i}$ be either $\left(2 \varepsilon_{i}, 4 \varepsilon_{i}\right)$ or $\left(4 \varepsilon_{i}, 2 \varepsilon_{i}\right), \varepsilon_{i}= \pm 1$. We define a pure 3-braid $\alpha\left[\varepsilon_{1} u_{1}, \varepsilon_{2} u_{2}, \ldots, \varepsilon_{p} u_{p}\right]$ by

$$
\alpha\left[\varepsilon_{1} u_{1}, \varepsilon_{2} u_{2}, \ldots, \varepsilon_{i} u_{i}, \varepsilon_{i+1} u_{i+1}\right]=\left(\alpha\left[\varepsilon_{1} u_{1}, \varepsilon_{2} u_{2}, \ldots, \varepsilon_{i} u_{i}\right]\right)\left(\varepsilon_{i+1} u_{i+1}\right),
$$

where we interpret $\alpha\left[\varepsilon_{1} u_{1}, \varepsilon_{2} u_{2}, \ldots, \varepsilon_{p} u_{p}\right]$ as $\alpha$ if $p=0$. Let $K_{\alpha\left[\varepsilon_{1} u_{1}, \varepsilon_{2} u_{2}, \ldots, \varepsilon_{p} u_{p}\right]}$ be the oriented 2-bridge knot with diagram $G_{\alpha\left[\varepsilon_{1} u_{1}, \varepsilon_{2} u_{2}, \ldots, \varepsilon_{p} u_{p}\right]}$, so $K_{\alpha}$ is $5_{2}$ in the table of [15]. Let $\mathscr{K}_{\alpha ; \varepsilon_{1}, \varepsilon_{2}, \ldots, \varepsilon_{p}}$ be the set of $2^{p}$ knots $K_{\alpha\left[\varepsilon_{1} u_{1}, \varepsilon_{2} u_{2}, \ldots, \varepsilon_{p} u_{p}\right]}$, $\varepsilon_{i} u_{i}=\left(2 \varepsilon_{i}, 4 \varepsilon_{i}\right)$ or $\left(4 \varepsilon_{i}, 2 \varepsilon_{i}\right)$, and $\mathscr{K}_{\alpha, p}$ be the union $\bigcup_{\varepsilon_{i}= \pm 1} \mathscr{K}_{\alpha ; \varepsilon_{1}, \varepsilon_{2}, \ldots, \varepsilon_{p}}$, so $\mathscr{K}_{\alpha, 0}=\left\{K_{\alpha}\right\}$. Then all the knots in $\mathscr{K}_{\alpha ; \varepsilon_{1}, \varepsilon_{2}, \ldots, \varepsilon_{p}}$ are skein-equivalent by [8, Propositions 3.1 and 3.2], and mutually nonisotopic by [8, Lemma 3.1]. All the knots in $\mathscr{K}_{\alpha, p}$ share the same $Q$ and Alexander polynomials by Lemmas 4 and 5. Since for a 2-bridge knot the minimal crossing number equals the maximal degree of the $Q$ polynomial plus one [11, 13], they have the same 
minimal crossing number, which we denote by $c_{\alpha, p}$, so $c_{\alpha, 0}=5$. We denote the Jones polynomial of $K_{\alpha\left[\varepsilon_{1} u_{1}, \varepsilon_{2} u_{2}, \ldots, \varepsilon_{p} u_{p}\right]}\left(\right.$ resp. $K_{\alpha}$ ) by $V_{\alpha ; \varepsilon_{1}, \varepsilon_{2}, \ldots, \varepsilon_{p}}(t)$ (resp. $\left.V_{\alpha}(t)=t-t^{2}+2 t^{3}-t^{4}+t^{5}-t^{6}\right)$ and the writhe of $G_{\alpha\left[\varepsilon_{1} u_{1}, \varepsilon_{2} u_{2}, \ldots, \varepsilon_{p} u_{p}\right]}$ (resp. $G_{\alpha}$ ) by $w_{\alpha ; \varepsilon_{1}, \varepsilon_{2}, \ldots, \varepsilon}\left(\right.$ resp. $w_{\alpha}=-6$ ). From Lemma 1 (ii), we have

$$
\begin{aligned}
& \left\langle G_{\alpha\left[\varepsilon_{1} u_{1}, \ldots, \varepsilon_{p} u_{p}, \varepsilon u\right]}\right\rangle \\
& =A^{6 \varepsilon}\left\langle G_{\alpha\left[\varepsilon_{1}, \ldots, \varepsilon_{p}\right]}\right\rangle\left\{A^{-16 \varepsilon}+A^{-8 \varepsilon}-1+\left(A^{-16 \varepsilon}-1\right)\left(A^{-8 \varepsilon}-1\right) d^{-2}\right. \\
& \left.\quad \times\left\langle G_{\alpha\left[\varepsilon_{1} u_{1}, \ldots, \varepsilon_{p} u_{p}\right]}\right\rangle\left\langle G_{\alpha\left[\varepsilon_{1} u_{1}, \ldots, \varepsilon_{p} u_{p}\right]} !\right\rangle\right\} .
\end{aligned}
$$

Because $V_{\alpha ; \varepsilon_{1}, \ldots, \varepsilon_{p}}\left(A^{4}\right)=\left(-A^{3}\right)^{-w_{\alpha ; \varepsilon_{1}, \ldots, \varepsilon_{p}}}\left\langle G_{\alpha\left[\varepsilon_{1} u_{1}, \ldots, \varepsilon_{p} u_{p}\right]}\right\rangle$ and $w_{\alpha ; \varepsilon_{1}, \ldots, \varepsilon_{p}}=w_{\alpha}-$ $4\left(\varepsilon_{1}+\cdots+\varepsilon_{p}\right)$, we have

$$
\begin{aligned}
V_{\alpha ; \varepsilon_{1}, \ldots, \varepsilon_{p}, \varepsilon}(t)=t^{\varepsilon} V_{\alpha ; \varepsilon_{1}, \ldots, \varepsilon_{p}}(t)\left\{t^{\varepsilon}+t^{3 \varepsilon}-t^{5 \varepsilon}\right. \\
\left.+\left(t^{\varepsilon}-1\right)^{2}\left(t^{2 \varepsilon}+1\right) V_{\alpha ; \varepsilon_{1}, \ldots, \varepsilon_{p}}(t) V_{\alpha ; \varepsilon_{1}, \ldots, \varepsilon_{p}}\left(t^{-1}\right)\right\} .
\end{aligned}
$$

Let $R_{\alpha ; \varepsilon_{1}, \ldots, \varepsilon_{p}}$ and $r_{\alpha ; \varepsilon_{1}, \ldots, \varepsilon_{p}}$ be the maximal and minimal degrees of $V_{\alpha ; \varepsilon_{1}, \ldots, \varepsilon_{p}}(t)$. Then $c_{\alpha, p}=R_{\alpha ; \varepsilon_{1}, \ldots, \varepsilon_{p}}-r_{\alpha ; \varepsilon_{1}, \ldots, \varepsilon_{p}}[10,14,16]$. It is easy to see that

$$
\begin{gathered}
R_{\alpha ; \varepsilon_{1}, \ldots, \varepsilon_{p}, \varepsilon}=R_{\alpha ; \varepsilon_{1}, \ldots, \varepsilon_{p}}+c_{\alpha, p}+3 \varepsilon+2, \text { and } \\
r_{\alpha ; \varepsilon_{1}, \ldots, \varepsilon_{p}, \varepsilon}=R_{\alpha ; \varepsilon_{1}, \ldots, \varepsilon_{p}}-2 c_{\alpha, p}+3 \varepsilon-2 .
\end{gathered}
$$

Thus we have $c_{\alpha, p+1}=3 c_{\alpha, p}+4=3^{p+1}\left(c_{\alpha, 0}+2\right)-2=7 \cdot 3^{p+1}-2$, and $R_{\alpha ; \varepsilon_{1}, \ldots, \varepsilon_{p}}=R_{\alpha}+\left(c_{\alpha, 0}+2\right)\left(3^{p}-1\right) / 2+3\left(\varepsilon_{1}+\cdots+\varepsilon_{p}\right)=\left(7 \cdot 3^{p}+5\right) / 2+$ $3\left(\varepsilon_{1}+\cdots+\varepsilon_{p}\right)$, which shows that at least $p+1$ polynomials in $V_{\alpha ; \varepsilon_{1}, \ldots, \varepsilon_{p}}(t)^{\prime} \mathrm{s}$ are distinct.

Remark 3. $\mathscr{K}_{\alpha, \varepsilon_{1}}=\left\{K_{\alpha\left(2 \varepsilon_{1}, 4 \varepsilon_{1}\right)}, K_{\alpha\left(4 \varepsilon_{1}, 2 \varepsilon_{1}\right)}\right\}, c_{\alpha, 1}=19, R_{\alpha ; \varepsilon_{1}}=3 \varepsilon_{1}+13$, $r_{\alpha ; \varepsilon_{1}}=3 \varepsilon_{1}-6$.

$$
\begin{gathered}
\mathscr{K}_{\alpha ; \varepsilon_{1}, \varepsilon_{2}}=\left\{K_{\alpha\left(2 \varepsilon_{1}, 4 \varepsilon_{1}\right)\left(2 \varepsilon_{2}, 4 \varepsilon_{2}\right)}, K_{\alpha\left(2 \varepsilon_{1}, 4 \varepsilon_{1}\right)\left(4 \varepsilon_{2}, 2 \varepsilon_{2}\right)},\right. \\
\left.K_{\alpha\left(4 \varepsilon_{1}, 2 \varepsilon_{1}\right)\left(2 \varepsilon_{2}, 4 \varepsilon_{2}\right)}, K_{\alpha\left(4 \varepsilon_{1}, 2 \varepsilon_{1}\right)\left(4 \varepsilon_{2}, 2 \varepsilon_{2}\right)}\right\},
\end{gathered}
$$

$c_{\alpha, 2}=65, R_{\alpha ; \varepsilon_{1}, \varepsilon_{2}}=34+3\left(\varepsilon_{1}+\varepsilon_{2}\right)$. We can see by a computer calculation that $V_{\alpha ; 1,-1}(t) \neq V_{\alpha ;-1,1}(t)$.

Link case. We change the definition of the pure 3 -braid $\alpha\left[\varepsilon_{1} u_{1}, \varepsilon_{2} u_{2}, \ldots, \varepsilon_{p} u_{p}\right]$ as follows:

$$
\alpha=S_{2}^{2} S_{1}^{2} S_{2}^{-2} ; \alpha\left[\varepsilon_{1} u_{1}, \ldots, \varepsilon_{i} u_{i}, \varepsilon_{i+1} u_{i+1}\right]=\beta S_{1}^{2 m} \beta^{-1} S_{1}^{2 n} \beta,
$$

where $\beta=\left[\varepsilon_{1} u_{1}, \ldots, \varepsilon_{i} u_{i}\right]$ and $\varepsilon_{i+1} u_{i+1}=(2 m, 2 n)$. Consider the oriented 2-

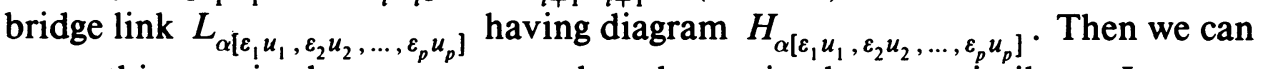
prove this case in the same way as the other, using lemmas similar to Lemmas 1-4 and Lemma 6. 
Remark 4. If $\varepsilon_{i} u_{i}=\left(2 \varepsilon_{i}, 2 \varepsilon_{i}\right)$ and $\alpha=S_{2}^{a_{1}} S_{1}^{b_{1}} \ldots S_{2}^{a_{n}} S_{1}^{b_{n}}, a_{i}, b_{i}= \pm 2$, then each set $\mathscr{K}_{\alpha ; \varepsilon_{1}, \ldots, \varepsilon_{p}}$ consists of a single fibered 2-bridge knot. See, for example, [6, Lemma 6.3]. Thus we can prove: There exist arbitrarily many fibered 2bridge knots (resp. links) with the same $Q$ and Alexander polynomials but mutually distinct Jones polynomials.

\section{REFERENCES}

1. R. D. Brandt, W. B. R. Lickorish, and K. C. Millett, A polynomial invariant for unoriented knots and links, Invent. Math. 84 (1986), 563-573.

2. G. Burde and H. Zieschang, Knots, De Gruyter Studies in Math., vol. 5, Walter de Gruyter, Berlin and New York, 1985.

3. J. H. Conway, An enumeration of knots and links, in Computational Problems in Abstract Algebra (J. Leech, ed.), Pergamon Press 1969, 329-358.

4. C. F. Ho, A new polynomial invariant for knots and links-preliminary report, Abstracts Amer. Math. Soc. 6 (1985), 300.

5. V. F. R. Jones, A polynomial invariant for knots via von Neumann algebras, Bull. Amer. Math. Soc. 12 (1985), 103-111.

6. T. Kanenobu, Examples on polynomial invariants of knots and links, Math. Ann. 275 (1986), 555-572.

7. __ Relations between the Jones and $Q$ polynomials for 2-bridge and 3-braid links, Math. Ann. 285 (1989), 115-124.

8. __ Examples on polynomial invariants of knots and links II, Osaka J. Math. 26 (1989), 465-482.

9. T. Kanenobu and T. Sumi, in preparation.

10. L. H. Kauffman, State models and the Jones polynomial, Topology 26 (1987), 395-407.

11. M. E. Kidwell, On the degree of the Brandt-Lickorish-Millet-Ho polynomial of a link, Proc. Amer. Math. Soc. 100 (1987), 755-762.

12. W. B. R. Lickorish and K. C. Millett, A polynomial invariant of oriented links, Topology 26 (1987), 107-141.

13. T. Miyauchi, On the highest degree of absolute polynomials of alternating links, Proc. Japan Acad. Ser. A 63 (1987), 174-177.

14. K. Murasugi, Jones polynomials of alternating links, Trans. Amer. Math. Soc. 295 (1986), 147-174.

15. D. Rolfsen, Knots and links, Math. Lecture Series no. 7, Publish or Perish, Berkeley, 1976.

16. M. B. Thistlethwaite, A spanning tree expansion of the Jones polynomial, Topology 26 (1987), 297-309.

Department of Mathematics, Kyushu University, Fukuoka 812, Japan 